\title{
Micetismo: Reporte de dos casos fatales y revisión bibliográfica
}

\section{Mycetism: Report of two fatal cases and review of the literature.}

Norma Castillo Godoy ${ }^{1}$, César Augusto Durán López ${ }^{2}$, Mireya Matamoros ${ }^{3}$

https://doi.org /10.5377/rcfh.v5i2.8918

${ }^{1}$ Unidad Médico-Legal del Hospital Escuela, ${ }^{2}$ Departamento de Patología, Universidad Nacional Autónoma de Honduras $^{3}$, Investigación y Docencia en Ciencias Forenses, Dirección de Medicina Forense, Ministerio Público de Honduras y Hospital San Felipe, Secretaria de Salud.

Correspondencia a:dileniagodoy@gmail.com

\section{CITAR COMO}

Castillo Godoy ND, Durán López CA, Matamoros M. Micetismo: Reporte de dos casos fatales y revisión bibliográfica. Rev. cienc. forenses Honduras. 2019; 5(2): 25-34.

Recibido: Marzo 2018, Aceptado: Junio 2019

Los autores declaran que no existe conflicto de interés en la publicación de este artículo.

\section{RESUMEN}

Justificación: El micetismo es la intoxicación producida por la ingestión de setas silvestres, que puede presentarse clínicamente con diferentes síndromes toxicológicos, dependiendo de la especie de hongo involucrada y sus toxinas. Su importancia médico legal radica en que la ingesta puede ocurrir de manera accidental, homicida o suicida. Objetivo: Revisar aspectos relevantes sobre el micetismo, para el reconocimiento de síndromes toxicológicos asociados a la ingesta de setas. Metodología: Se revisaron diferentes bases de datos utilizando las palabras clave: micetismo, Amanita phalloides, autopsia médico legal, abordaje diagnóstico. Resultados: $\underline{\text { A phalloides, }}$ contiene amatoxinas causantes de insuficiencia hepática aguda y las falotoxinas responsables de manifestaciones gastrointestinales 10 horas después de la ingesta, característica del síndrome tóxico faloidiano. Los casos que se describen corresponden a dos femeninas de la misma familia, una mujer adulta 36 años embarazada y su hija de seis años, procedentes de una comunidad rural en la parte central de Honduras, que fallecieron después de la ingestión de setas silvestres y cuyas autopsias se realizaron en la Dirección de Medicina Forense de Tegucigalpa, para establecer la causa de muerte. En la historia médico legal se consignó que los hongos fueron cocinados por la madre y consumidos por ella y su hija. Conclusión: Es recomendable la capacitación continua de los médicos de emergencia en hospitales, para el reconocimiento de síndromes toxicológicos asociados a la ingesta de setas, con el propósito de tratar oportunamente estos pacientes y ofrecerles mejor oportunidad de sobrevivir.

\section{PALABRAS CLAVE}

Micetismo, Amanita phalloides, Autopsia, Abordaje terapéutico, Reporte de caso, Revisión Bibliográfica.

\section{ABSTRACT}

Justification: Mycetism is the poisoning caused by the ingestion of wild mushrooms, which can occur clinically with different toxicological syndromes, depending on the species of fungus involved and its 
toxins. Its medicolegal importance is that the intake can occur accidentally, homicidally or suicidally. Objective: To review relevant aspects of mycetism, for the recognition of toxicological syndromes associated with mushroom intake.

Methodology: Different databases were reviewed using the keywords: mycetism, Amanita phalloides, medicalegal autopsy, diagnostic approach. Results: A. phalloides, contains amatoxins that cause acute liver failure and falotoxins wich are responsible for gastrointestinal manifestations 10 hours after intake, characteristic of the phalloidin toxic syndrome. The cases described correspond to two women from the same family, a 36-year-old pregnant woman and her 6-year-old daughter, from a rural community in the central part of Honduras, who died after ingestion of wild mushrooms and whose autopsies were performed at the Department of Medicina Forense of Tegucigalpa, to establish the cause and manner of death. In the medicolegal history, it was reported that the mushrooms were cooked by the mother and consumed by her and her daughter. Conclusion: Continuous training of emergency doctors in hospitals is recommended for the recognition of toxicological syndromes associated with mushroom intake, to treat these patients on time and offer them a better chance of survival.

\section{KEYWORD}

Mushroom poisoning, Amanita phalloides, Autopsy, Treatment, Case report, Bibliographic review.

\section{INTRODUCCIÓN}

El micetismo se refiere a la intoxicación causada por el consumo de setas (macromicetos) ${ }^{1,2}$, común en varios países incluyendo Honduras, y según la Organización Mundial de la Salud se presenta con alta tasa de mortalidad dependiendo de la especie involucrada ${ }^{3-6}$.

En España, el Servicio de Información Toxicológica del Instituto Nacional de Toxicología y Ciencias
Forenses reportó que la incidencia anual es de 5 a 10 casos por millón de habitantes, de los cuales el $30 \%$ son por amatoxinas, con mortalidad aproximada del $7 \%$ entre pacientes tratados y $30 \%$ entre los no tratados ${ }^{1,3}$.

En Honduras, el micetismo se presenta de manera cíclica y circunscrita a zonas donde se consumen setas tradicionalmente durante la época lluviosa ${ }^{3-}$ 5 .

La intoxicación por setas tiene importancia médico legal ya que la ingesta puede ocasionar la muerte de manera accidental, homicida o suicida, y en relación a la posible responsabilidad profesional, cuando el médico no considera u obvia su diagnóstico para el adecuado tratamiento del paciente.

El micetismo puede ocurrir accidentalmente en adultos por la confusión de setas tóxicas con comestibles debido a su similitud, como se muestra en la Figura 1. También puede ocurrir en jóvenes que tienen el hábito de ingerir setas alucinógenas en combinación con alcohol, marihuana u otras drogas de abuso y en niños que ingieren setas que crecen en el jardín o césped de guarderías.

Se conocen entre 50 a 100 tipos de setas venenosas para los humanos, principalmente del género amanita, siendo las especies importantes $\underline{A}$. phalloides, $\underline{A}$. virosa, y $\underline{A}$. verna; responsables de la mayoría de intoxicaciones, reportándose a Amanita phalloides como la especie que causa elevada mortalidad ${ }^{2}$.

El objetivo de este trabajo es revisar aspectos relevantes sobre el micetismo, para el reconocimiento de síndromes toxicológicos asociados a la ingesta de setas, con el propósito de tratar oportunamente estos pacientes y ofrecerles mejor oportunidad de sobrevivir.

Entre las toxinas que posee $\underline{A}$. phalloides, están las amatoxinas que causan insuficiencia hepática y la 
falotoxina, responsable del inicio tardío (> 10horas) de vómitos y diarrea. Otras especies como $\underline{\text { Amanita }}$ $\underline{\text { smithiana contiene una nefrotoxina. La Amanita }}$ muscaria y Amanita pantherina contienen toxinas de isoxazol que principalmente causan alteraciones del estado mental. También se han descrito otros géneros de setas que no son amanitas pero contienen amatoxinas como Lepiota, Galerina y Conocybe. ${ }^{7,8,9,10,11}$.

\section{METODOLOGÍA DE BÚSQUEDA}

Se realizó una búsqueda en pubmed, google académico, science direct, usando los términos: Micetismo, Amanita phalloides, Autopsia, Abordaje terapéutico.

\section{Descripción general de las amatoxinas y propiedades}

Las amatoxinas se consideran las toxinas más importantes de las setas venenosas por estar involucradas en el $90 \%$ de las intoxicaciones letales. Las amatoxinas son liposolubles y termoestables, no se descomponen, ni desnaturalizan cuando se congelan, cuecen o procesan; pueden provocar la muerte de un adulto a dosis de $0,1 \mathrm{mg} / \mathrm{kg}$ de peso corporal. La ingestión de 20 a 25 g de $\underline{A}$. phalloides implicaría la exposición a 5-8 mg de amatoxina, letal para un adulto de $70 \mathrm{~kg}$. Los niños se consideran un grupo especialmente sensible a estas intoxicaciones, presentando la mortalidad más elevada ${ }^{8,12}$.

Su absorción ocurre rápidamente en el intestino y son transportadas al hígado, en los hepatocitos inhiben la ARN polimerasa, alterando la transcripción de ARN mensajero necesario para la síntesis de proteínas, produciendo daño celular caracterizado morfológicamente por necrosis hepá tica centrolobular y clínicamente por la aparición insidiosa de insuficiencia hepática ${ }^{9,11}$.

\section{Progresión clínica del Micetismo}

La progresión clínica de la intoxicación por setas

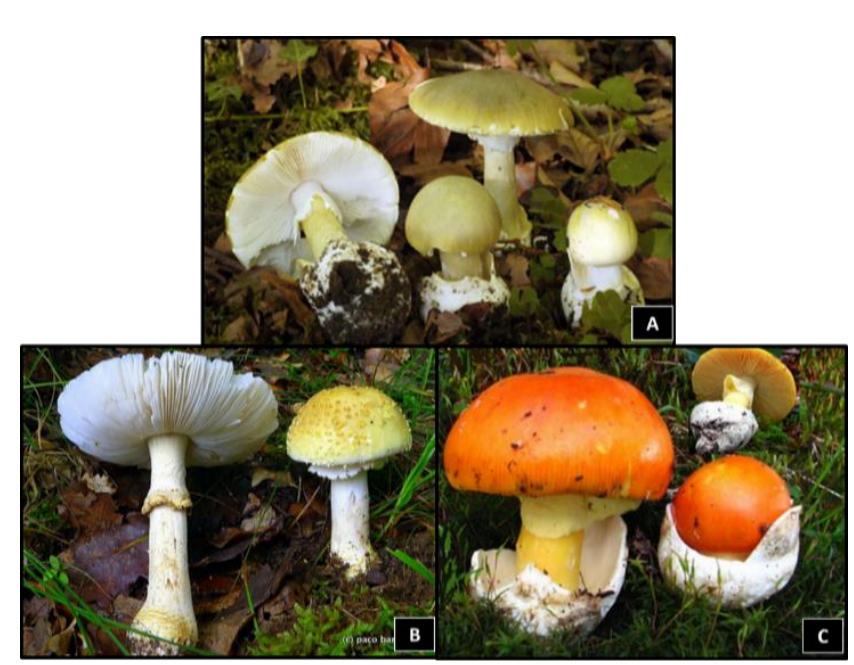

Figura 1. A. Amanita phalloides tiene un sombrero color verde, que puede volverse amarillento y después blanquecino, en la parte inferior de mismo se ven láminillas blancas. El pie es blanco con manchas verdes diminutas. Su carne es blanca, tierna, inolora en ejemplares jóvenes y maloliente, agria y repugnante en especimenes viejos. B: Amanita junquillea var. amici, algunos micólogos desaconsejan su consumo por la posible confusión con $\underline{A}$. phalloides. C: $\underline{\text { Amanita }}$ caesárea, hongo conocido como "choro" y considerada delicia culinaria en el Occidente de Honduras. Fotografías tomadas de: http://www.fichasmicologicas.com/?micos=1\&alf=A\&

puede ser dividida en cuatro fases ${ }^{13}$ :

1. Latencia (asintomática): Las toxinas como tal no tienen propiedades irritativas y por tanto no hay síntomas.

2. Gastrointestinal: Dura en promedio de 1 a 24 horas, hay náuseas, dolor abdominal, diarrea que puede llegar a ser sanguinolenta, deshidratación, oliguria y alteraciones electrolíticas.

3. Remisión clínica (mejoría aparente o convaleciente): Ocurre entre 36 y 48 horas después de la ingesta, las pruebas de laboratorio suelen estar alteradas y puede presentarse ictericia.

4. Falla hepática y/o falla renal: Empiezan a elevarse las aminotransferasas, hay aumento de la LDH, aumento de la bilirrubina, alteración de la 
coagulación, hipoglucemia, encefalopatía hepática e insuficiencia renal.

Al final de estas cuatro etapas puede presentarse falla multiorgánica con coagulación intravascular diseminada, acidosis metabólica, infarto de miocardio, trombosis mesentérica, convulsiones y finalmente la muerte entre una y tres semanas después de la ingesta o la resolución favorable con rápida mejoría de la función hepática y renal ${ }^{13}$.

\section{Tratamiento}

Aunque algunos autores mencionan que no existe antídoto para la intoxicación por setas, en estudios de intoxicación experimental, algunos fármacos como las cefalosporinas y penicilinas, han demostrado tener una acción protectora por la saturación del sistema multiespecífico de transporte en la membrana del hepatocito, impidiendo la entrada de las amatoxinas ${ }^{3}$.

El tratamiento médico está orientado a tratar de eliminar las toxinas del tracto digestivo, control de líquidos y electrolitos, la administración de penicilina o ceftriaxona, atención de problemas específicos como convulsiones, falla hepática y el mantenimiento de la diuresis. Así entre las medidas para el manejo y tratamiento de la intoxicación por setas se puede enumerar ${ }^{7}$ :

1. Control de la vía aérea y ventilación mecánica en casos necesarios.

2. Monitorización continua del balance hídrico, presión venosa central y parámetros analíticos como medición de electrolitos y $\mathrm{pH}$.

3. Aporte nutricional.

4. Reposición de líquidos con solución salina y soluciones glucosadas.

5. Evitar uso de benzodiacepinas.

6. Colocar sonda nasogástrica o nasoduodenal con carbón activado seriado, a razón de $1 \mathrm{~g} / \mathrm{kg}$ de peso la primera dosis, seguido de una dosis de algún catártico osmótico y después carbón activado a $0,5 \mathrm{~g} / \mathrm{kg}$ de peso cada 4 horas por 72 horas.

7. En pacientes que hayan convulsionado o tengan riesgo de convulsionar, o estén en coma y previamente intubados, se recomienda un procedimiento controvertido para eliminar las amatoxinas que consiste en administrar carbón activado a través de sonda gastroduodenal, cerrar la sonda por dos horas y después aspirar continuamente el contenido digestivo por dos horas.

8. Penicilina $\mathrm{G} 300,000 \mathrm{UI} / \mathrm{Kg} /$ día (en infusión continua), con ajuste de dosis si hay insuficiencia renal.

9. Mantener diuresis forzada de $4-5 \mathrm{ml} / \mathrm{kg} / \mathrm{h}$ usando algún diurético como furosemida o manitol para lograr la eliminación del $80 \%$ de la amanitina en las primeras 2 a 3 horas. Se añade furosemida con el objetivo de conseguir diuresis de 200 $\mathrm{ml} /$ hora en las primeras 24 a 48 horas.

10. Si hay insuficiencia hepática administrar Nacetil-cisteína en bolo de $150 \mathrm{mg} / \mathrm{kg}$ intravenoso, en 1 hora. Continuar con 50 mg/kg en 4 horas y 100 $\mathrm{mg} / \mathrm{kg}$ en infusión continua durante 16 horas.

11. Vitamina K $10 \mathrm{mg} /$ día, intravenosa.

12. Técnicas de depuración extracorpórea: Se han comunicado algunos casos con resultados favorables utilizando la hemodiálisis con cartuchos de carbón activado, la plasmaféresis y la diálisis de albúmina.

\section{Diagnóstico diferencial}

A través del síndrome toxicológico observado en los pacientes, se puede determinar el tipo de setas involucrado, haciendo la asociación entre el periodo de latencia y los síntomas, como se ilustra en la Figura 2.

\section{PRESENTACIÓN DE CASOS}

Se describen dos casos de la misma familia, correspondientes a mujer adulta, de 36 años 
Figura 2. Diagnóstico diferencial según la cronología y clínica de la intoxicación por setas

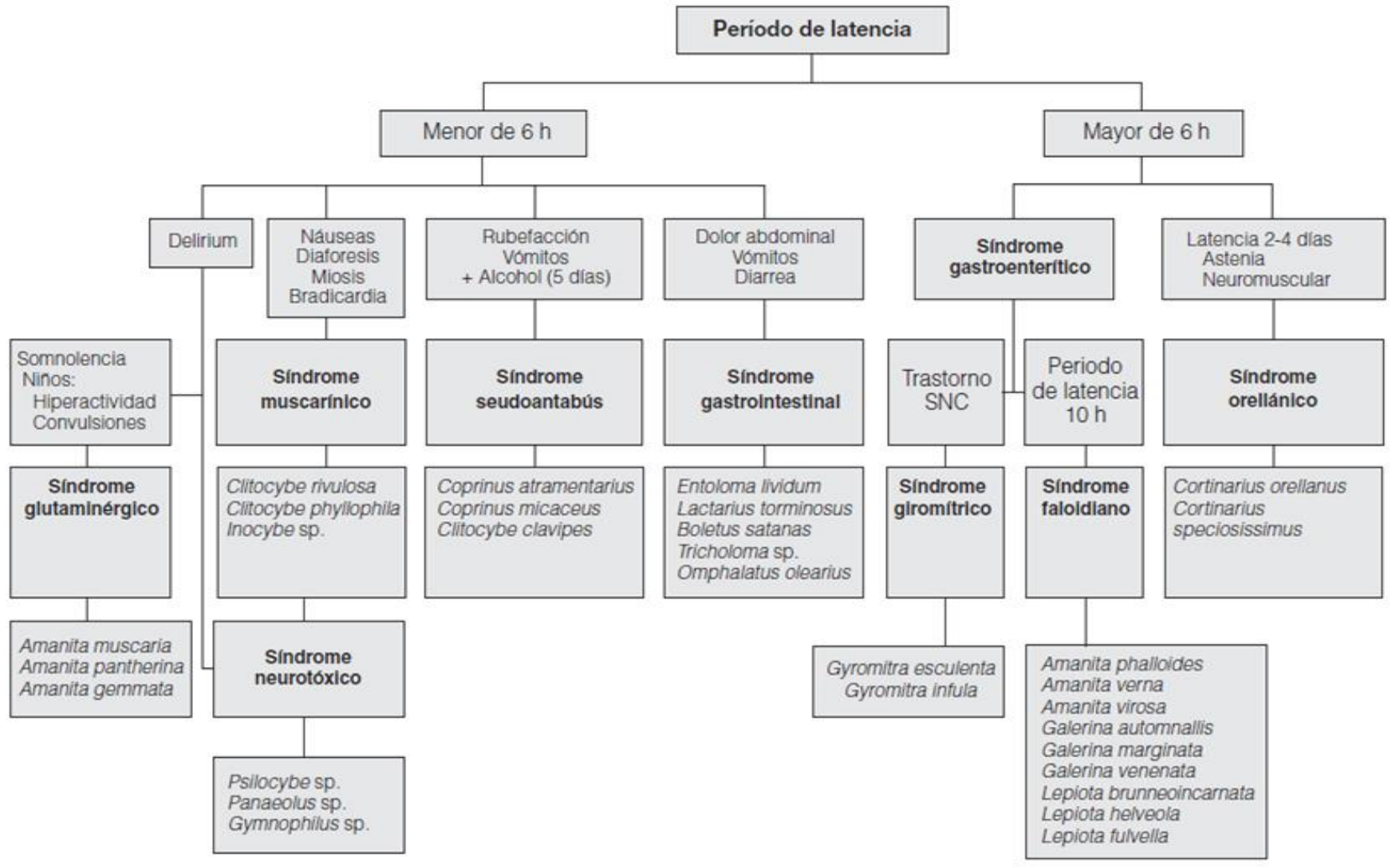

Tomado de Benítez Macías JF. y col. en: Intoxicaciones agudas por setas. Algoritmo diagnóstico de las intoxicaciones agudas por setas ${ }^{14}$

embarazada y su hija de seis años, procedentes de una comunidad rural en la parte central de Honduras, sin antecedentes personales patológicos, que fallecieron después del consumo de setas silvestres y cuyas autopsias se realizaron en la Dirección de Medicina Forense de Tegucigalpa.

La historia médico legal consignó que los hongos fueron cocinados por la madre y consumidos por ella y su hija.

El Cuadro 1 detalla las características clínicas de ambos casos y la Figura $\mathbf{3}$ esquematiza la línea temporal desde la ingesta de los hongos silvestres y el desarrollo de las etapas clínicas hasta la muerte.
Debido a que durante la estancia hospitalaria no fue posible determinar la causa de muerte, las fallecidas fueron remitidas para autopsia médico legal; el Cuadro 2 muestra el resumen de los hallazgos más relevantes de la autopsia.

\section{DISCUSIÓN}

La ingesta de hongos silvestres es tradicional en la región occidental de Honduras ${ }^{17}$. Para el consumo humano se requiere la habilidad de reconocer las especies tóxicas. Los casos descritos proceden de la parte central del país, región donde no es habitual comer setas, sin embargo, de acuerdo a información no corroborada la madre fallecida era de La Esperanza (Occidente de Honduras), lugar donde el consumo de setas es tradicional ${ }^{17}$. 
CUADRO 1. Presentación de las características clínicas de los casos.

\begin{tabular}{|c|c|c|}
\hline & CASO 1 (Femenina de 36 años, madre) & CASO 2 (Femenina de 6 años, hija) \\
\hline Fase de latencia & Tres (3) días & 13 horas \\
\hline Síntomas en la fase gastrointestinal & $\begin{array}{l}\text { Dolor epigástrico intenso, vómitos, diarrea, } \\
\text { puño percusión positiva. }\end{array}$ & Dolor epigástrico intenso, vómito, disentería. \\
\hline $\begin{array}{l}\text { Centro Médico que atendió el inicio } \\
\text { del cuadro clínico }\end{array}$ & Hospital Regional. & Hospital Regional. \\
\hline Diagnósticos de Ingreso & $\begin{array}{l}\text { 1. Embarazo de } 253 / 7 \mathrm{SG} \times \mathrm{FUM} \text {. } \\
\text { 2. Síndrome gastroentérico de etiología } \\
\text { bacteriana con deshidratación leve. } \\
\text { 3. Pielonefritis. }\end{array}$ & No disponibles, no fue posible obtenerlos. \\
\hline Tratamiento inicial & $\begin{array}{l}\text { Antibioticoterapia (Ceftriaxona). } \\
\text { Hidratación intravenosa. }\end{array}$ & $\begin{array}{l}\text { Antibioticoterapia no especificada } \\
\text { Hidratación intravenosa. }\end{array}$ \\
\hline $\begin{array}{l}\text { Evolución clínica después de la fase } \\
\text { gastrointestinal }\end{array}$ & Inestabilidad hemodinámica. & Mejoría al 4 ㅇ́á. \\
\hline $\begin{array}{l}\text { Síntomas y signos más severos } \\
\text { (transición entre la fase } \\
\text { gastrointestinal y falla multiorgánica) }\end{array}$ & $\begin{array}{l}\text { - Vómitos en pozo de café. } \\
\text { - Deshidratación. } \\
\text { - Alteración de la conciencia. } \\
\text { - Taquicardia. } \\
\text { - Disminución de pulsos periféricos. } \\
\text { - Cianosis y frialdad distal. } \\
\text { - Hematuria. }\end{array}$ & $\begin{array}{l}\text { - Vómitos y diarrea de tres días de evolución. } \\
\text { - Hematemesis. } \\
\text { - Fiebre. } \\
\text { - Convulsiones. } \\
\text { - Hipoglicemia. } \\
\text { - Deterioro neurológico. } \\
\text { - Sangrado por sonda nasogástrica y tubo } \\
\text { endotraqueal. Sangrado masivo nariz, recto y } \\
\text { sitios de venopunción. }\end{array}$ \\
\hline $\begin{array}{l}\text { Diagnósticos de referencia a Hospital } \\
\text { Nacional }\end{array}$ & $\begin{array}{l}\text { Sangrado digestivo alto, falla hemodinámica, } \\
\text { óbito fetal y falla multiorgánica. }\end{array}$ & $\begin{array}{l}\text { Descartar neuroinfección, status convulsivo } \\
\text { secundario a neuroinfección e hipoglicemia }\end{array}$ \\
\hline $\begin{array}{l}\text { Diagnóstico de ingreso a Hospital } \\
\text { Nacional }\end{array}$ & $\begin{array}{l}\text { 1. Sangrado digestivo alto con inestabilidad. } \\
\text { hemodinámica de etiología a investigar. } \\
\text { 2. Sospecha de intoxicación por hongos. } \\
\text { 3. Embarazo de } 275 / 7 \text { por USG con } \\
\text { sospecha de óbito fetal. }\end{array}$ & $\begin{array}{l}\text { 1.-Falla ventilatoria secundaria. } \\
\text { 2.-Choque hipovolémico/séptico. } \\
\text { 3.-Coagulación intravascular diseminada por } \\
\text { clínica } \\
\text { 4.-Intoxicación alimentaria. } \\
\text { 5.-Síndrome convulsivo febril complejo. }\end{array}$ \\
\hline Tratamiento en Hospital Nacional & Omeprazol, Ceftriaxona, Clindamicina. & $\begin{array}{l}\text { Vitamina K, Ceftriaxone, Diazepam por convulsión, } \\
\text { Fenitoína, Infusión Midazolam/Fentanilo, Infusión } \\
\text { de dopamina. }\end{array}$ \\
\hline $\begin{array}{llll}\text { Condiciones } & \text { asociadas } & \text { a } & \text { la } \\
\text { intoxicación. } & & & \end{array}$ & $\begin{array}{l}\text { - Trastorno hemorrágico: Sangrado } \\
\text { Digestivo alto con inestabilidad } \\
\text { hemodinámica. } \\
\text { - Falla multiorgánica. }\end{array}$ & $\begin{array}{l}\text { - Hiperglicemia. } \\
\text { - Trastornos hidroelectrolíticos: Hipocalcemia e } \\
\text { hipokalemia. } \\
\text { - Trastorno hemorrágico: Sangrado orofaríngeo } \\
\text { que procedía de vía digestiva, sangrado rectal y } \\
\text { por sitios de venopunción. } \\
\text { - Paro cardiorrespiratorio (se realizó reanimación } \\
\text { cardiopulmonar). }\end{array}$ \\
\hline $\begin{array}{l}\text { Diagnósticos consignados antes de la } \\
\text { muerte }\end{array}$ & $\begin{array}{l}\text { 1. Embarazo } 28 \text { SG x FUM con óbito fetal } \\
\text { 2. Sangrado digestivo alto de etiología a } \\
\text { investigar. } \\
\text { 3. Sospecha de intoxicación por hongos. } \\
\text { 4. shock séptico foco de entrada } \\
\text { gastrointestinal. }\end{array}$ & $\begin{array}{l}\text { 1. Síndrome posparada con sospecha de muerte } \\
\text { cerebral. } \\
\text { 2. Falla multiorgánica. } \\
\text { 3. Choque séptico/Hemorrágico. } \\
\text { 4. Síndrome Convulsivo Secundario. } \\
\text { 5. Coagulación Intravascular Diseminada: Sangrado } \\
\text { digestivo alto y epistaxis. } \\
\text { 6. Necrosis hepática. } \\
\text { 7. Falla renal aguda. } \\
\text { 8. Sospecha intoxicación por amanita. }\end{array}$ \\
\hline $\begin{array}{l}\text { Inicio de tratamiento por sospecha de } \\
\text { Intoxicación por setas }\end{array}$ & Ninguno. & $\begin{array}{l}\text { cuarto día posterior a la ingesta, } \\
\text { Piperacilina/tazobactam, Omeprazol, Sucralfato, N- } \\
\text { acetilcisteína e hidrocortisona. }\end{array}$ \\
\hline Exámenes de laboratorio & $\begin{array}{l}\text { Hemoglobina } 11.2 \mathrm{~g} / \mathrm{dl} \text {. Plaquetas } 59 \times 103 \mathrm{c} \\
\text { /mm3, BUN } 8 \mathrm{mg} / \mathrm{dl} \text {, Creatinina } 1.53 \mathrm{mg} / \mathrm{dl} \text {, } \\
\text { Albumina: } 2.7 \mathrm{~g} / \mathrm{dl} \text {, Bilirrubina total: } 6.8 \\
\mathrm{mg} / \mathrm{dl} \text {, Bilirrubina directa: } 5.0 \mathrm{mg} / \mathrm{dl} \text {. Sodio: } \\
148 \mathrm{mg} / \mathrm{dl} \text {. }\end{array}$ & $\begin{array}{l}\text { Tiempos de protrombina mayor de } 90 \mathrm{seg} \text {, tiempo } \\
\text { control de } 12.3 \text { seg. Hemoglobina } 4.2 \mathrm{~g} / \mathrm{dl} \text {. } \\
\text { Plaquetas } 158,000 \times 103, \mathrm{c} / \mathrm{mm} 3, \mathrm{BUN} 6 \mathrm{mg} / \mathrm{dl} \text {, } \\
\text { creatinina } 1.3 \mathrm{mg} / \mathrm{dl} \text {, AST: } 4155 \mathrm{U} / \mathrm{l}, \mathrm{LDH}: 1998 \mathrm{U} / \mathrm{L} \text {, } \\
\text { albumina: } 1.5 \mathrm{~g} / \mathrm{dl} \text {, Bilirrubina total: } 2.8 \mathrm{mg} / \mathrm{dl} \text {. }\end{array}$ \\
\hline de setas hasta el fallecimiento. & 6 días. & 6 días. \\
\hline
\end{tabular}


Figura 3. Esquema temporal de las etapas clínicas del micetismo que presentaron los casos

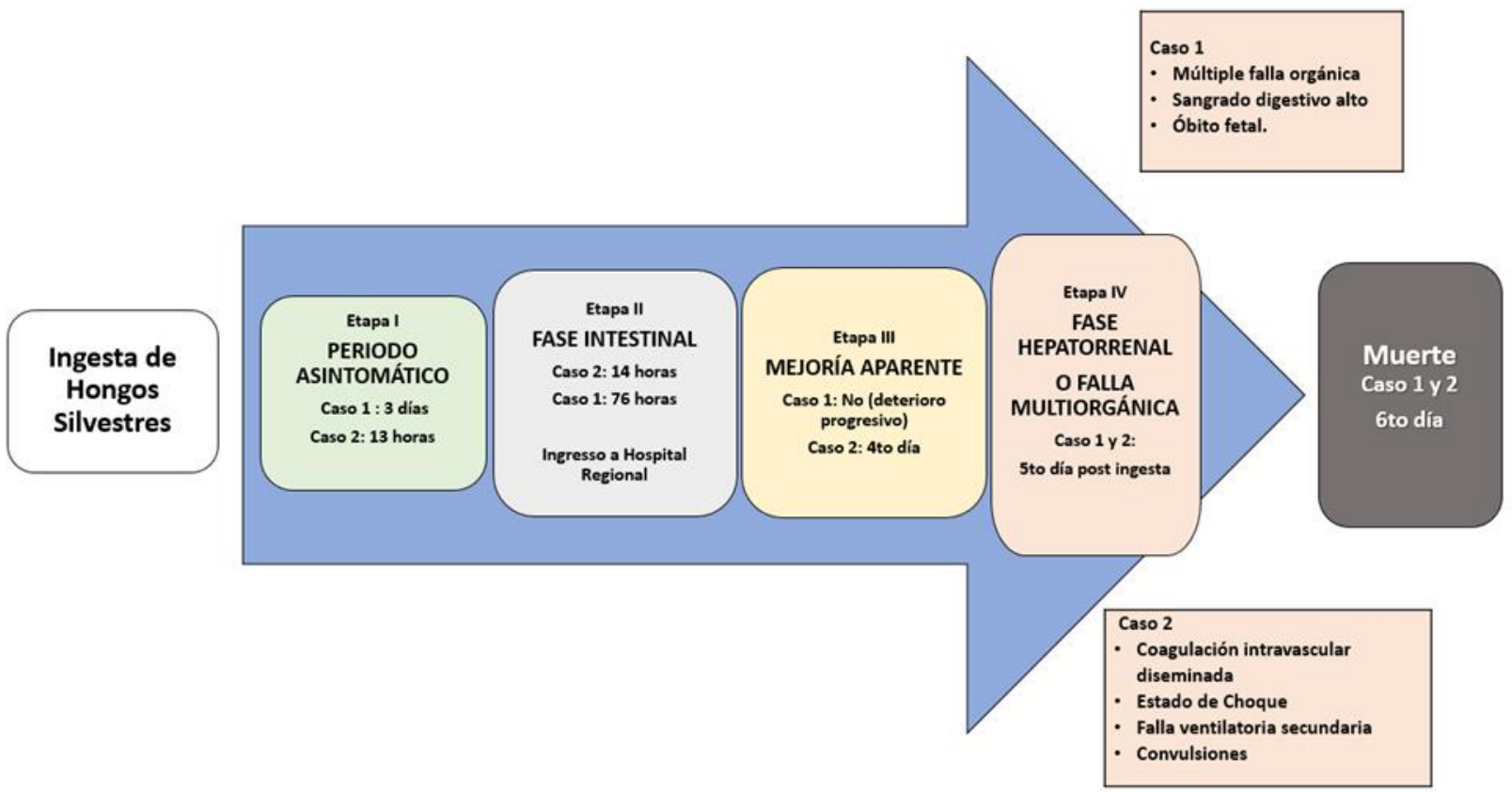

CUADRO 2. Hallazgos en la autopsia médico legal

HALLAZGOS

CASO № 1 (MADRE) CASO № 2 (HIJA)

\section{Examen externo del cadáver}

- Ictericia generalizada.

- Cianosis en labios y uñas.

Examen interno del cadáver
Derrame pleural bilateral.

Ascitis.

Congestión visceral generalizada.

- $\quad$ Estomago conteniendo líquido color en poso de café y congestión de mucosa estomacal e intestinal.

Producto de la gestación con muerte intrauterina.
- Edema cerebral

- Edema laríngeo

- Mucosa traqueal congestiva

\section{Reporte de \\ Histopatología forense}

- $\quad$ Hígado con necrosis hemorrágica multifocal.

- $\quad$ Encefalopatía hipóxica.

- Necrosis tubular aguda

Congestión visceral generalizada.

Hemorragia reciente difusa en submucosa de estómago y colon.

No disponible.
Necrosis isquémica difusa en la mucosa colónica.

- Esteatosis hepática severa.

\section{Laboratorio toxicológico de muestras postmortem}

Causa de muerte

Manera de muerte Insuficiencia hepática fulminante secundaria a intoxicación alimentaria por setas.
Accidental. 
Las intoxicaciones por setas se pueden clasificar según la duración de la etapa asintomática en:

1.-Intoxicaciones con período de latencia breve (el aparecimiento de los síntomas se considera temprano), inferior a seis horas, variando entre 30 minutos y tres o cuatro horas, por lo general son intoxicaciones leves.

2.- Intoxicaciones con periodo de latencia largo (el aparecimiento de los síntomas se considera tardío), superior a seis horas, variando entre nueve $y$ quince horas, ocasionalmente llegando hasta los diez o quince días, en su mayoría son intoxicaciones graves ${ }^{1,3,14}$. Los dos casos mostrados presentaron período de latencia largo, y mostraron los síntomas característicos de la fase intestinal: nausea, vómito y diarrea, característicos del síndrome faloidiano producido por la falotoxinas $3,7,9,14,18$.

En los presentes casos, después de la sintomatología intestinal la menor mostró la fase de mejoría, la madre no, en su lugar ella tuvo deterioro progresivo hasta la falla multiorgánica, Generalmente, después que los síntomas gastrointestinales mejoran, el hígado se deteriora rápidamente, con incremento de las transaminasas y caída de los factores de coagulación $3,7,14$.

Para el quinto día ambas tenían falla multiorgánica, cuya aparición varía entre tres y seis días, caracterizada por falla hepática fulminante, encefalopatía hepática, insuficiencia renal aguda prerrenal y edema cerebral, generalmente este último es la causa directa de muerte en el $80 \%$ de los casos $3,7,14$; sin embargo, en estos casos no se evidenció herniación cerebral en la autopsia.

\section{CONCLUSIONES}

En estos casos no fue posible detectar la toxina debido a la falta de sospecha clínica temprana, sumado a que en el nivel regional de atención no se dispone de este tipo de análisis de laboratorio, sin embargo, pese que no se demostró la toxina en fluidos biológicos, ni en los alimentos porque no se recibieron muestras de las setas consumidas; basándose en los hallazgos de autopsia y en la información clínica como: el periodo de latencia largo, síntomas gastrointestinales, el desenlace fatal y el hallazgo en la histopatología de necrosis hepática multifocal ${ }^{19,20}$, se consideró que la causa básica de muerte fue intoxicación por ingestión de setas toxicas, posiblemente atribuidas al género amanita y la causa directa insuficiencia hepática con trastorno hemorrágico.

La manera de muerte se estableció como accidental, ya que se consignó en la historia médico legal la ingesta de las setas cocinadas por la madre y no se obtuvieron datos de la investigación policial, que evidenciaran una hipótesis alternativa.

En relación al tratamiento empleado, debido a que no se sospechó tempranamente la intoxicación por setas, las medidas fueron únicamente de sostén. Hasta el cuarto día, en la fase de falla hepática, se sospechó que la menor podía estar presentando un cuadro clínico de intoxicación por amanitas, decidiéndose la administración de piperacilina/tazobactam, N Acetil-cisteína y Vitamina K.

No se tomaron medidas para reducir la reabsorción del tóxico en el tracto digestivo, pudiéndose haber intentado ciclos de eliminación de toxinas mediante la colocación de sonda nasoduodenal y administración de Carbón activado, seguido de algún catártico osmótico. Tampoco se administró Penicilina G para bloquear la captación de amatoxina por los hepatocitos.

\section{ASPECTOS MEDICO LEGALES}

En la evaluación médico forense del micetismo es importante la recolección minuciosa de indicios físicos, químicos y biológicos, su protección en cadena de custodia tiene como objetivo obtener toda la información necesaria para el sustento científico que permita vincular la historia clínica y 
los hallazgos de autopsia al tóxico, posibilitando determinar la causa de la muerte.

\section{RECOMENDACIONES}

Por el alto riesgo para la vida que implica la recolección empírica y la ingesta accidental de hongos, se podrían realizar campañas de educación continua en la población donde el consumo de setas es tradicional, para que esta práctica sea más segura y se preserve para fines turísticos.

Se sugiere capacitar continua y sistemáticamente a los médicos de emergencia en hospitales regionales y nacionales en los departamentos del centro y occidente de Honduras, para el reconocimiento de síndromes toxicológicos asociados a la ingesta de setas, con el propósito de tratar oportunamente estos pacientes y ofrecerles mejor oportunidad de sobrevivir.

\section{REFERENCIAS BIBLIOGRAFICAS}

1. Salud Madrid. Subdirección de Gestión y Seguimiento de Objetivos en Hospitales. Guía de actuación ante sospecha de intoxicación por setas - micetismos. [Internet]. [citado 2016 Octubre 04]. Disponible en:

http://www.madrid.org/bvirtual/BVCM017323.pd $\underline{f}$

2. Graeme, Kimberlie A. Mycetism: A Review of the Recent Literature J Med Toxicol. [Revista en internet] 2014 Jun; [ citado 2017 nov 06].10(2): 173-189.

Disponible

en:

http://www.reviberoammicol.com/199916/121125.pdf

3. Josep Piqueras-Carrasco. Intoxicaciones por setas, una actualización. Rev Esp Med Legal. [Internet] 2014[ citado 2016 Julio 07];40(1):19 - 29. Disponible en: http://ac.elscdn.com/S0377473213000369/1-s2.0s0377473213000369-main.pdf? tid=a521da5e9091-11e68d300000aabOf01\&acdnat=1476286769 f617095 9a8150d9a3b310c0cf48c8047

4. Sarmiento E, Fontecha G. Conocimiento tradicional de los hongos en el occidente de Honduras. Revista Ciencia y Tecnología. [Internet] 2013. [Citado 2016
Octubre 12]13; Disponible en: file://C:/Users/user1/Downloads/1710-6105-1PB.pdf

5. Organización Mundial de la Salud. Unintentional poisoning: burden of disease Data by country. [ Portal en Internet $\{$ Citado 2017 nov 06\}. disponible en:

http://apps.who.int/gho/data/node.main.SDGPOI SON393

6. Fichas micológicas, página de internet (consultado nov 208) Disponible en http://www.fichasmicologicas.com/?micos=1\&alf =A\&art=24

7. Arif Alper CEVIK, Ilhami UNLUOGLU. Factors Affecting Mortality and Complications in Mushroom Poisonings Over a 20 Year Period: A Report from Central Anatolia. Turk J Emerg Med. [Internet]. 2016 [ citado 2017 Octubre 04]; 14(3):104-108. Disponible en: https://www.ncbi.nlm.nih.gov/pubmed/27331181

8. Pérez Gordillo J H, Colbert Rodríguez M, Cruz Rivera N, Pérez Gordillo G. Falla hepática fulminante por Intoxicación por Amanita Verna. Reporte de tres casos. Rev Hosp Jua Mex [Internet] 2009 [ citado 2016 Oct 02]; 76(3): 168-175. Disponible en: www.medigraphic.com/pdfs/juarez/ju2009/ju093k.pdf

9. Juliana Garcia, et al. Amanita phalloides poisoning: Mechanisms of toxicity and treatment. Food and Chemical Toxicology. [Revista en Internet]. December 2015, [ Citado 2017 nov 07] Volume 86, Pages 41-55. Disponible en: http://ac.elscdn.com/S0278691515300594/1-s2.0-

S0278691515300594main.pdf? tid=53d2f27c909 211e68b3200000aab0f6b\&acdnat=1476287062 1 2e4e85778a291c64e5ffad94deef1a8

10. Díaz JH. Amatoxin-Containing Mushroom Poisonings: Species, Toxidromes, Treatments, and Outcomes Wilderness \& Environmental Medicine [ Revista en Internet] 2018 [ citado 2018 abril 04] 29(1), 111-118. Disponible en: https://ac.elscdn.com/S1080603217302752/1-s2.0S1080603217302752-main.pdf? tid=30551886eb4f-48aa-83da-

3aae48fc531d\&acdnat $=15231223160$ 0e8ffb90e23 98fc4d68972f60b19bcf

11. Horowitz BZ, Gossman WG. Toxicity, Mushrooms, Amatoxin. [2017]. en: StatPearls [Internet]. Treasure Island (FL): StatPearls Publishing; 2018 
Jan-. Disponible en: https://www.ncbi.nlm.nih.gov/books/NBK43105 2l

12. Yilmaz I, Ermis F, Akata I, Kaya E. A Case Study: What Doses of Amanita phalloides and Amatoxins Are Lethal to Humans? Wilderness Environ Med [Revista en Internet]. 2015 Dec [ citado 2018 abril 04]; 26(4):491-6. Disponible en: https://www.researchgate.net/publication/2827 32741 A Case Study What Doses of Amanita phalloides and Amatoxins Are Lethal to Huma $\underline{\text { ns }}$

13. Barquero, M; Bolaños P. Falla hepática aguda y otros trastornos causados por el consumo de Amanita phalloides. Med. leg. Costa Rica [Internet]. 2016 [citado 2017 Oct 11] ; 33( 1 ): 96-102. Disponible en: https://www.scielo.sa.cr/scielo.php?script=sci ar ttext\&pid=S1409-00152016000100096

14. Benítez Macías J F, García Gil D, Brun Romero FM y Nogué Xarau N. Intoxicaciones agudas por setas. Rev Clin Esp. [Internet]. 2009 [citado 2017 Octubre 04];209(11):542-549. Disponible en:file:///C:/Users/user1/Downloads/S00142565 09730619 S300 es\%20(1).pdf

15. Secretaría de Salud. Centro de Información Toxicológica de Veracruz. "Guía de diagnóstico y tratamiento de intoxicación por setas" (Micetismo). [Internet], [ citado 2018, Abril 05] Disponible en: https://www.ssaver.gob.mx/citver/files/2018/03 Micetismo.pdf
16. Ventura, $S$, Ruiz $C$, Durán $E$, Mosquera $M$, Bandrés F, Campos F, Queraltó JM (2015). Amanitinas Revista del Laboratorio Clínico, 8 (3), 109-126. doi: 10.1016 / j.labcli.2015.05.001scihub.se/10.1016/j.labcli.2015.05.001

17. Así de lindo se celebró el festival del choro en la Esperanza, tomado de Honduras It's great, disponible en https://hondurasisgreat.org/lafestival-choro2018/

18. Rodríguez-Maldonado $M$, López-Álvarez $F$, Andrade Padilla M. Intoxicación por hongos: A propósito de un niño con falla hepática. Rev Mex Pediatr [Internet] 2008 [ citado 2017 Octubre 04]; 75(4): 171-174. Disponible en: http://www.medigraphic.com/pdfs/pediat/sp2008/sp084g.pdf

19. Sánchez-Guzmán S. Falla hepática fulminante secundaria a intoxicación por hongos (setas) reporte de caso y revisión. Rev Med MD 2016; 7.8 (1) Disponible en: https://www.medigraphic.com/pdfs/revmed/md2016/md161e.pdf

20. Alamoni et al. Intoxicación por Amanita phalloides, diagnóstico y tratamiento. Arch. argent. pediatr. [Internet]. 2006 Ago [citado 2018 Abr 05]; 104( 4 ): 372-374. Disponible en: http://www.scielo.org.ar/scielo.php?script=sci a rttext\&pid=S0325-00752006000400018\&lng=es. 